\title{
Symposium on free radicals
}

\section{October 1992 \\ Institut Pasteur, Paris, France}

\author{
Organizing committee \\ M. Chignard \\ G. Grondeux \\ B. B. Vargaftig
}

Scientific committee

\author{
M. Audrain \\ L. Baud \\ M. Chignard \\ B. Descamps-Latscha \\ R. Fauve \\ B. B. Vargaftig
}




\section{Programme}

\section{Session I}

Chairmen:

A. Favier (Grenoble, France) and B. Halliwell (London, UK)

Introduction

B. B. Vargaftig (Paris, France)

Biochemistry of free radicals

M. Fontecave (Grenoble, France)

Production of free radicals. Antioxidant mechanisms

R. V. Bensasson (Paris, France)

Free radicals and tissue injuries

B. Halliwell (London, UK)

\section{Session II}

Chairmen:

R. V. Bensasson (Paris, France) and P. A. Ward (Ann Arbor, USA)

Artificial antioxidants: interest, evaluation on experimental models and limits on their uses

A. Favier (Grenoble, France)

Genetic modulation of brain antioxidant defense capacity: transgenic mice overexpressing copper-zinc superoxide dismutase as models for the study of free radicals metabolism and ageing

I. Ceballos-Picot (Paris, France) - abstract available (C1)

The biology of free radicals and their roles in pathology

P. A. Ward (Ann Arbor, USA)

Free radicals and their role in chronic synovitis

S. E. Edmonds (London, UK) - abstract available (C2)

\section{Session III}

Chairmen:

J. M. Carney (Lexington, USA) and B. Descamps-Latscha (Paris, France)

Reactive forms of oxygen and inflammation

J. Hakim (Paris, France)

Free radicals and cytokines: key stones of immune dysfunction in chronic uremia and bemodialysis?

B. Descamps-Latscha (Paris, France)

Free radicals and CNS diseases

J. M. Carney (Lexington, USA)

Superoxide radical and nitric oxide mediate neuronal injury after bypoxia in cortical neurone cultures

C. Cazevieille (Montpellier, France)-abstract available (C3) 


\section{Session IV}

\section{Chairmen:}

C. Auclair (Villejuif, France) and B. B. Vargaftig (Paris, France)

Oxidative damages induced by carcinogenic and antineoplastic agents

C. Auclair (Villejuif, France)

$\mathrm{H}_{2} \mathrm{O}_{2}$ increase, catalase activity impairment, DNA repair-deficiency and carcinogenesis

M. Vuillaume (Paris, France) - abstract available (C4)

Free radicals and cardiovascular diseases

J. Y. Artigou (Paris, France)

Polynuclear activation during angioplasty

C. Pasquier (Paris, France) — abstract available (C5)

Free radicals and critical care

C. Franssen (Liège, Belgium)

Conclusion 
The GREMI (Groupe de Recherches et d'Etudes sur les Médiateurs de l' Inflammation, Institut Pasteur, 25 Rue du Dr Roux,75015 Paris; Fax: +331 456 88703) is one of the French Societies promoting research in the field of inflammation. Among other activities, the GREMI organises seminars and symposia and one was recently held at the Institut Pasteur, Paris, initiated by G. Grondeux on Free Radicals. We thought that it would be of interest to the general readership of Mediators of Inflammation so we have put together abstracts from this meeting printed in the order in which they were presented.

2 March 1993

Paris

B. Boris Vargaftig 
C1

\section{Genetic modulation of brain antioxidant defense capacity. Transgenic mice overexpressing copper-zinc superoxide dismutase as models for the study of free radicals metabolism and ageing}

\section{Ceballos-Picot, A. Nicole and P. M. Sinet}

Laboratoire de Biochimie Génétique, CNRS URA 1335, Hôpital Necker, 149 rue de Sevres, 75015 Paris, France

Oxidants are ubiquitous in our aerobic environment and could play an etiological role in ageing and neurodegenerative diseases such as Alzheimer's disease. All cells contain several antioxidant enzymes, most importantly, superoxide dismutases (MnSOD and CuZnSOD), glutathione peroxidase (GPX), glutathione reductase (GR) and catalase. The individual contribution of these antioxidant enzymes in neuronal protection during ageing and under in vivo conditions remain unknown.

The aim of our study was firstly to obtain a comprehensive profile of the brain antioxidant defense potential and peroxidative damage during ageing in control mice. A positive correlation between age and activities of CuZnSOD $(r=0.47$; $p<0.01)$ and GPX $(r=0.72 ; p<0.0001)$ was observed. CuZnSOD and GPX activity are independently regulated during brain ageing: temporal changes of these two enzymes do not correlate. No modification in MnSOD activity and basal lipid peroxidation was observed as a function of age. Secondly we used genetic manipulations to construct transgenic mice that specifically overexpress $\mathrm{CuZnSOD}$ in order to understand the role of $\mathrm{CuZnSOD}$ in neuronal ageing. The human transgene expression resulted in 1.9-fold increased CuZnSOD activity in the brain, was stable during ageing and resulted in enhanced rate of basal lipid peroxidation and in increased MnSOD activity in the three age groups studied (2,12 and 28 months). Other antioxidant enzymes (GPX and GR) did not exhibit modifications indicating the independence of the regulation between CuZnSOD and glutathionerelated enzymes probably due to their different cellular localization in the brain. Immunohistochemical and in situ hybridization analysis of brain sections revealed that human CuZnSOD and mRNA were preferentially expressed in neurons, particularly in the stratum pyramidale of Ammon's horn throughout the CA1-CA4 fields of the hippocampus and in the granule cell layer of the dentate gyrus and was absent or weak in the remaining layers

\section{C2}

\section{Free radicals and their role in chronic synovitis}

\author{
S. E. Edmonds, K. Fairburn, R. B. Williams, \\ G. A. Ellis, N. B. Nazhat and D. R. Blake
}

The Inflammation Group, The London Hospital Medical College, London E1 1AD, UK

Given that inflammation has a natural tendency to settle, the chronicity of the synovitis that characterizes rheumatoid arthritis (RA) presents an interesting conundrum. We argue that the persistence of the synovitis relates to the unusual characteristics of a moving diarthrodial joint. Once inflammation has become established, the joint becomes hypoxic and yet, paradoxically, generates reactive oxygen species (ROS).

Using a fine polarographic needle electrode (Eppendorf) we have measured the $\mathrm{pO}_{2}$ of rheumatoid synovial tissue and compared it to normal synovium. The $\mathrm{pO}_{2}$ is shifted downward in RA with pockets of tissue having $\mathrm{O}_{2}$ tensions of between 5 and $15 \mathrm{mmHg}$. Exercising rheumatoid synovium lowers the $\mathrm{pO}_{2}$ in the joint cavity even further.

The rheumatoid synovial cavity has a positive intra-articular pressure (IAP) at rest. This is in contrast to the normal joint where the resting pressure is mildly sub-atmospheric. When the rheumatoid joint is exercised, the IAP rises even further and the synovial microvasculature is temporarily cut off. We have demonstrated this in a number of joints including the knee and metacarpophalangeal. In this way, the hypoxia is compounded and leads to the stimulation of calcium uptake by synovial cells which in turn activates phospholipase $\mathrm{A}_{2}$.

Following exercised-induced microvascular occlusion, the blood supply is restored (reperfusion) and ROS are generated. Electron spin resonance and spin trapping have been used to detect the ROS that are produced. Using DBNBS as the spin trap, a characteristic three line spectrum is formed when synovial tissue is rendered transiently hypoxic and then reperfused. The three-line spectrum is assigned to one-electron peroxidatic oxidation of DBNBS leading to a nitroxyl-type radical.

Within the joint, such ROS can damage a variety of critical biomolecules and stimulate or damage selective cell populations. We have demonstrated that proteins (including $\operatorname{IgG}, \alpha_{1}$-antitrypsin and cytoskeleton proteins), lipoproteins, lipids, carbohydrates and glycosaminoglycans all undergo attack by ROS.

Mediators of Inflammation · Vol 2 1993 
The innervation of the joint may also be damaged, resulting in the loss of neurogenic feedback loops and the subsequent abolition of the protective mechanism of reflex muscle inhibition. This, we believe, allows for the generation of the high IAP that are observed in inflamed joints.

Oxygen free radicals are also known to stimulate cultured fibroblasts to proliferate and may therefore contribute to pannus formation in rheumatoid arthritis. Hydrogen peroxide inhibits proteoglycan synthesis by cultured bovine articular cartilage and we have recently demonstrated that this molecule is a potent stimulator of osteoclastic bone resorption. This may have an influence on the development of the erosive bone changes seen in RA.

The antioxidant capacity of the inflamed joint is impaired. Ascorbate levels are low in RA synovial fluid and most of this essential antioxidant is in the oxidized dehydroascorbate form. The latter may indicate consumption of other antioxidants and indeed we have demonstrated evidence for the depletion of $\alpha$-tocopherol within the rheumatoid joint. This suggests a role for the use of antioxidant therapy in the treatment of chronic joint disease.

\section{C3}

\section{Superoxide radical and nitric oxide mediate neuronal injury after hypoxia in cortical neurone cultures}

\author{
C. Cazevieille, A. Muller and C. Bonne
}

\author{
Laboratoire de Physiologie Cellulaire, Faculté de \\ Pharmacie, Université Montpellier I, France
}

Ischaemia provokes neuron injury and the release of excitotoxic amino acids e.g. glutamate. Nitric oxide synthesized under glutamate receptor stimulation participates in neuronal damage in vivo and in vitro. In the same way, by using rat cortical neuron in culture we demonstrated that inhibition of nitric oxide synthesis with $N^{G}$-nitro-L-arginine, was able to protect cells from hypoxia/reoxygenation-induced toxicity. Superoxide dismutase and D,L-2-amino-5-phosphonovaleric acid, an antagonist of glutamate receptors also protect the cells. These results suggest the sequential involvement of the superoxide anion generation, the release of excitatory amino acids and the synthesis of nitric oxide in hypoxia/reoxygenation-related neurotoxicity.

\section{C4}

\section{$\mathrm{H}_{2} \mathrm{O}_{2}$ increase, catalase activity impairment, DNA repair-deficiency and carcinogenesis}

\author{
M. Vuillaume, ${ }^{1}$ L. Daya-Grosjean, ${ }^{2}$ \\ E. Quéinnec, ${ }^{1}$ B. Dutrillaux ${ }^{3}$ and A. Sarasin ${ }^{2}$
}

${ }^{1}$ URA 686 CNRS, ENS, 46 rue d'Ulm 75230 Paris, Cedex 05, France; ${ }^{2}$ UPR 42, Villejuif; ${ }^{3}$ Laboratoire de Cytogénétique, CEA, Fontenay-aux-Roses, France

Xeroderma pigmentosum (XP) and trichothiodystrophy (TTD) are two human autosomal recessively-transmitted diseases characterised by DNA repair deficiency. Trichothiodystrophy is not a cancer-prone disease. Therefore, unpaired UVinduced lesions do not appear to be enough to give rise to tumours. The aim of our research was concerned with the fundamental mechanisms and their clinical applications.

In 21 different XP diploid fibroblast lines, catalase activity was decreased by five compared to controls, while XP heterozygote lines exhibited intermediary responses. All seven TTD lines were as deficient in UV-induced lesion repair as XP cell strains and exhibited a high level of catalase activity. $\mathrm{UV}$-irradiation induces five times more $\mathrm{H}_{2} \mathrm{O}_{2}$ compared with TTD or controls. Two results indicated that:

(i) catalase decrease and $\mathrm{H}_{2} \mathrm{O}_{2}$ increase are not in direct relation with the loss of one allele $11 \mathrm{p}$,

(ii) molecular analysis of monomer for catalase transcription (only the tetramer form of enzyme is biologically active) showed no difference between normal, XP and TTD lines.

Our work is now engaged in:

(i) catalase deficiency reparation by in situ, enzymatic overexpression, and/or $\mathrm{H}_{2} \mathrm{O}_{2}$ scavengers addition;

(ii) studies of possible defect in catalase tetramerisation or absense of co-factors in the four active sites of catalase (ex vivo micro-biophysical investigations, cell by cell); (iii) ex vivo $\mathrm{H}_{2} \mathrm{O}_{2}$ measurements by enzymatic ultra-micro-electrodes ( $5 \mu$ diameter), cell by cell. (Programme Ultimatech) 
C5

\section{Neutrophils activation during angioplasty}

\author{
C. Pasquier, G. Steg, R. Gourgon and \\ J. Hakim
}

INSERM U. 294 CHU X, Bichat, Paris, France

Percutaneous transluminal coronary angioplasty (PTCA) represents injury to the coronary arterial wall and a model of transient ischaemia. Both of these processes can activate both circulating neutrophils and the complement system, which may participate in local stimulation of vascular smooth muscle proliferation, in post-ischaemic myocardial dysfunction, as well as in susceptibility to vasospasm at atherosclerotic sites.

The aim of this study was to demonstrate that PTCA could result in activation of the neutrophils and the complement system, as assessed ex vivo from tests on coronary sinus blood. Neutrophil activation was assessed by: (1) measurement of plasma lactoferrin (LF); (2) whole blood chemiluminescence stimulated in vitro by formylmethionyl-leucyl-phenylalanine, phorbol myristate and opsonized zymosan; (3) Plasma fluorescent thiobarbituric acid-reactive substances (TBA-RS). These measurements were done on blood drawn from the coronary sinus, before and immediately after PTCA (seven patients). To ensure that the changes observed after PTCA were not solely related to the arteriographic procedure, seven patients undergoing coronary arteriography were also studied.

After PTCA, LF increased two-fold $(p<0.02)$ whereas after arteriography a nonsignificant increase of $45 \%$ from baseline values was observed. Plasma TBA-RS did not change significantly in both groups. Whole blood chemiluminescence stimulated in vitro increased after PTCA, regardless of the stimulus used, while the increase was not significant after arteriography. Neutrophil count and adherence properties were not modified by either PTCA or arteriography. Total hemolytic complement $\left(\mathrm{CH}_{50}\right), \mathrm{C}_{3}, \mathrm{C}_{4}$ and $\mathrm{B}$ factor decreased slightly (7-16\%) after PTCA and after arteriography.

In conclusion, early after PTCA, the neutrophil oxidative response, as assessed by stimulated whole blood chemiluminescence, is increased, suggesting a 'priming' effect of PTCA on neutrophils. In addition, an increase in plasma lactoferrin is seen, indicating neutrophil activation. Finally, there is a mild global activation of the complement system, most likely related to the contrast agent infused during PTCA, and which may play a role in the 'priming' process. Thus, after PTCA, there are signs of activation of neutrophil and the complement system. 
P1

\section{Radiolysis study of sulfarlem, a sulfured antioxidant, in aerated linoleic acid micelles}

\author{
P. Ruimy-Ifrah, D. Jore, M. O. Christen ${ }^{1}$ \\ and C. Ferradini
}

Laboratoire de Chimie-Physique, URA 400 CNRS Université René Descartes, 45 rue des Saints-Pères F-75270, Paris Cedex 06; ' 1 Solvay Pharma Laboratoires de Thérapeutique Moderne (LTM France) BP 22,

F-92151, Suresnes, France

Sulfarlem (ADT) is a dithiol-thione derivative, the scavenging properties of which have been studied by steady-state radiolysis in a micellar system.

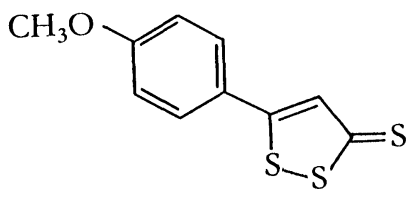

The micellar medium consists of linoleic acid (LH) $\left(10^{-2} \mathrm{~mol} \mathrm{l}\right)$ in water. $\gamma$ irradiations of such a medium leads to the formation of $\mathrm{L}^{*}$ free radicals according to:

$$
\mathrm{LH}+\mathrm{OH}^{\cdot} \rightarrow \mathrm{L}^{\cdot}+\mathrm{H}_{2} \mathrm{O}
$$

In the presence of oxygen, $\mathrm{L}$ affords the corresponding $\mathrm{LO}_{2}$ peroxyl radical:

$$
\mathrm{L}^{\cdot}+\mathrm{O}_{2} \rightarrow \mathrm{LO}_{2}^{*}
$$

In the absence of ADT, $\gamma$ irradiation results in a degradation of $\mathrm{LH}$ characterised by a decrease of the absorption at $270 \mathrm{~nm}$ and the formation of conjugated dienes observed at $234 \mathrm{~nm}$.

In the presence of ADT, its concentration increasing from $10^{-5}$ to $1.25 \times 10^{-4} \mathrm{moll}$, the experimental data indicate:

- a consumption of ADT (measured at $428 \mathrm{~nm}$ ) proportional to the absorbed dose.

- a degradation of LH and a formation of conjugated dienes lower than in the absence of ADT.

AD'T seems therefore capable of playing an efficient antioxidant role by scavenging the radical species involved in lipid peroxidation phenomena.
P2

\section{Photo-activable drugs with therapeutic potential}

\author{
D. Brault, ${ }^{1}$ C. Vever-Bizet, ${ }^{1}$ \\ B. Aveline, ${ }^{1}$ R. Bensasson, \\ M. Grandadam ${ }^{2}$ and D. Ingrand ${ }^{3}$
}

${ }^{1}$ Laboratoire de Biophysique, Muséum Nat Hist. Nat INSERM U201, CNRS URA 481, Paris; ${ }^{2}$ Laboratoire de Virologie, CERVI, Hôpital de la Pitié-Salpêtrière, Paris; ${ }^{3}$ Laboratoire de Bactériologie-Virologie-Immunologie, Hôpital Robert Debré, Reims, France

Photochemotherapy is being developed for tumour treatment and also shows promising potential for virus inactivation. This method involves the photosensitised generation of reactive intermediates, in particular singlet oxygen, which reacts with biological components leading to deleterious damages. The molecular mechanisms relevant to this new methods are examined with emphasis on processes induced by photosensitisers, such as porphyrin compounds, which present affinity for membrane systems. As shown by laser flash experiments using singlet oxygen luminescence detection, this species reacts with various components present in membranes. A promising application of these processes is the photoinactivation of viruses with a lipidic envelope such as the herpes virus and the human immunodeficiency virus (HIV). Interestingly, mutants resistant to usual antiviral drugs are also inactivated. The development of more efficient photosensitisers, in particular those presenting increased absorption of light in the red is likely to induce a breakthrough in these new approaches.

\section{P3}

\section{Isoprostaglandins: a new radical pathway of arachidonic acid metabolism}

\section{B. Rondot, T. Durand, J. C. Rossi, J. P. Girard and J. Rokach}

Université de Montpellier I; URA CNRS no.1.111. Faculté de Pharmacie, 15 avenue C. Flahault, 34060, Montpellier Cedex, France

Arachidonic acid (AA) is rapidly metabolised by several enzymatic systems leading to many 


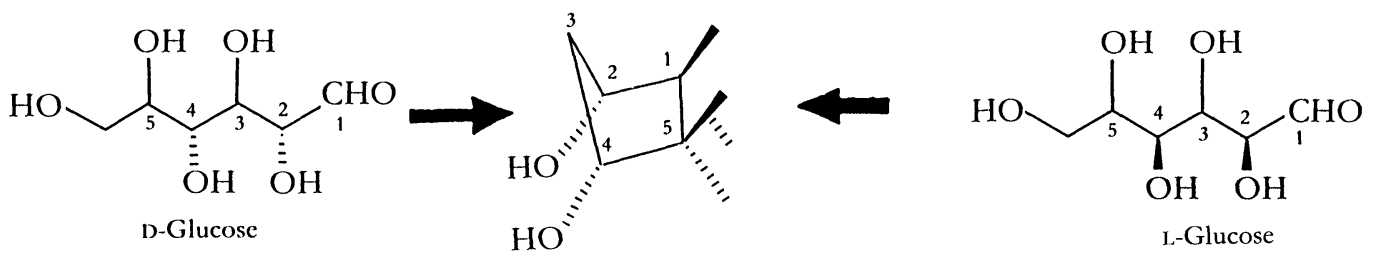<smiles>[R]C1[C@H]([R2])[C@H](O)C[C@H]1O</smiles><smiles>[R]=C=CC=CCCCC(=O)O</smiles>

FIG 1. Synthesis of IsoPGs

oxygenated metabolites. Recently, a series of $\mathrm{PGF}_{2 \alpha}$-like compounds (isoprostaglandins) with a potent biological activity were reported to exist in vivo in humans by a free radical catalysed mechanism.

If non-esterified AA is the substrate of cyclooxygenase systems, this non-enzymatic process may act on the esterified AA phospholipids. The formation of these phospholipid species in lipid bilayers may contribute in an important way to alterations in structure and function of cellular membranes, a well known point of oxidant stress.

To check this non-enzymatic process as an important pathway of AA metabolism, we have undertaken the total synthesis of 8-epi and 12-epi-PGF - $_{2 \alpha}$ (IsoPGs). The starting material is the D- and L-glucose (Fig. 1).

\section{P4}

\section{Specific and non-enzymatic determination of glutathione in biological mixtures of mercaptans}

\section{J.-C. Yadan, N. Aguini and J. Chaudiere}

Centre de Recherche Bioxytech, 2 adv des coquelicots, 94385 Bonneuil/Marne Cedex, France

The ubiquitous tripeptide glutathione $(\mathrm{GSH})$ is the most important biological mercaptan whose homeostasis plays a central role in most aerobic cells. Our aim was to develop a new colorimetric method for the measurement of GSH which would be easy to use, fast and specific. A large number of halogeno- $N$-alkylquinolinium structures were assessed as potential and chromogenic reagents of mercaptans (RSH).

Substitution of good leaving groups, in the 4position on the quinoline ring, was always characterised by fast kinetics and intense absorbance of the thioether adducts in the $340-370 \mathrm{~nm}$ range, around neutral $\mathrm{pH}$. For instance, the reaction of $0.05-10 \mathrm{mM}$ GSH with 4-chloro-7-trifluoromethyl-1-methyl-quinolinium (BXT-03015) in tenfold excess, is completed in less than $5 \mathrm{~min}$ at $\mathrm{pH}$ 7.3, and it yields a 4-thioetherquinolinium with strong absorbance at $356 \mathrm{~nm}$. In biological samples, a mixture of different mercaptans yields thioether adducts which cannot be resolved by means of colorimetry. In most cases however, fast HPLC separation of such adducts provides a convenient tool for measurement of all mercaptans.

When the GSH-adduct of BXT-03015 is further incubated at $\mathrm{pH}>13$ and ambient temperature, a fast reaction of $\beta$-elimination yields the corresponding 4-mercaptoquinolinium with strong absorbance around $400 \mathrm{~nm}$. In a survey of more than 20 other mercaptans, including mercaptoethanol, dithiothreitol, cysteine derivatives and various drugs, $\beta$-elimination was never observed in the above conditions. The latter, therefore, provides a chemical signature of GSH.

This new method requires only one sampling and two colorimetric determinations and it allows a fast reproducible and specific measurement of both total mercaptans and GSH in biological samples. 
P5

\section{Collecting and analysing procedures for pentane determination in alveolar air breath as a predictive factor of oxidative diseases and therapeutic agents}

\section{Massias, ${ }^{1}$ E. Postaire, ${ }^{1}$ C. Regnault, G. Rousset, ${ }^{2}$ P. Gossioux ${ }^{2}$ and G. Hazebroucq ${ }^{1}$ \\ ${ }^{1}$ Department of Clinical Pharmacy, School of Pharmacy, rue J. B. Clément, F 92290 \\ Châtenay-Malabry, France: ${ }^{2}$ INOCOSM, 43 Bd \\ Maréchal Joffre, F 92340 Bourg-la-reine, France}

Approximately 200 compounds have been detected in human breath, some of which have been correlated to various diseases. Formation of free radicals and lipid peroxidation are involved in many conditions including cellular damage. They are produced during normal metabolism and in pathological states. The peroxidation of polyunsaturated fatty acids, i.e. linolenic acid, which are cellular membrane compounds, induced pentane formation in pulmonary air breath. The determination of breath alkane is considered to be a valuable and elegant method to assess lipid peroxidation.

It is claimed that the collection and analysis of breath is a nontraumatic, easily repeated, and a useful noninvasive alternative to blood analysis. Our sampling system was designed to maximise analyte concentrations while minimising interference from exogenous contamination. The volatile compounds were captured on graphitised carbon and a molecular sieve, eluted from the trap by thermal desorption, and then assayed by gas chromatography with flame ionisation detection. The method is sensitive, linear and reproducible.

This collecting procedure was found to be well accepted by human volunteers and the breathcollecting apparatus was readily transported to sites outside the laboratory for the collection of sample. A study was performed with ten healthy volunteers. The average concentration of pentane found in alveolar breath samples was $4.336 \pm 1.67 \mathrm{nmol} / 1$.

\section{P6}

\section{A sensitive and specific method for measuring MDA in urine with an application to burned patients}

\author{
M. Guichardant, L. Valette-Talbi and \\ C. Cavadini
}

Nestec Ltd, Research Centre Vers-chez-les-Blanc, Post Office Box 44, 1000 Lausanne 26, Switzerland
Malondialdehyde (MDA) is one of the most studied products of lipid peroxidation. It is excreted in urine and is an indicator of lipid peroxidation in the diet and/or formed in the tissues.

The classical thiobarbituric acid (TBA) test has been widely used to measure MDA. MDA-TBA chromogens were isolated and quantified by HPLC. However in our hands, with biological samples such as urine or food, the chromatographic resolution was not adequate. To eliminate these interfering MDA-TBA chromogens which had spectra overlapping, we have modified this classical method. TBA reagent was replaced by 1,3-diethyl2-thiobarbituric acid (DETBA). The chromogen MDA-DETBA, less polar than MDA-TBA was easily isolated by solid phase extraction using C18 Bakerbond cartridges and then quantified by HPLC without interference at picomole levels using a fluorescent detector. The complete methodology and results concerning MDA in urine, from burned and traumatized patients, was presented.

\section{P7}

\section{Fast and specific assay of SOD activity based on accelerated autoxidation of a novel polycyclic catechol}

M. Moutet, C. Fargues, P. Huet, J. Xu, J.-C. Yadan and J. Chaudiere

Centre de Recherche Bioxytech, 2 av des coquelicots, 94385 Bonneuil/Marne Cedex, France

It is now widely recognized that the control of reactive oxygen species plays a major role in the biochemistry of aerobic organisms. Dismutation of superoxide $\left(\mathrm{O}_{2}^{-}\right)$into hydrogen peroxide and molecular oxygen is catalysed by superoxides dismutases (SODs) which are major protective enzymes. Much attention has been focused on the pharmalogical development and applications of SODs as therapeutic agents. The need for a convenient and reliable method of SOD activity assay, standardised and suitable for large series of biological samples, led us to develop a new assay of SOD activity.

This method is based on the SOD-mediated increase in the rate of autoxidation of BXT-01050 (I) in aqueous alkaline solution.<smiles>Oc1ccc2c(c1)CCC1Cc3cc(O)c(O)cc3C21</smiles> 
This autoxidation yields a chromophore with a maximal absorbance wavelength of $525 \mathrm{~nm}$. Two mechanisms of autoxidation are proposed. The first one involves two reversible steps of one-electron transfer to oxygen, producing two equivalents of $\mathrm{O}_{2}^{*-}$. Subsequent deprotonation of the resulting orthoquinone yields the chromophoric product. The second hypothesis is consistent with a one-electron oxidation of BXT-01050 into the corresponding semiquinone, which upon deprotonation of its five-membered ring, adds oxygen to form a tertiary peroxyl radical which then extrudes superoxide. In either case, equilibrium of $\mathrm{O}_{2}^{--}$ production are driven to the right by SOD activity. The assay is performed at $\mathrm{pH} 8.8,37^{\circ} \mathrm{C}$, in $50 \mathrm{mM}$ air-saturated 2-amino-2-methyl-1,3-propanediol buffer, containing $3 \mathrm{mM}$ boric acid and $0.1 \mathrm{mM}$ DTPA. The kinetic measurement of $525 \mathrm{~nm}$ absorbance is performed upon addition of BXT-01050, for $1 \mathrm{~min}$. This reagent is stabilized in stock solution acidified at $\mathrm{pH} 1.5$. The SOD activity is determined from the $\mathrm{V}_{\mathrm{s}} / \mathrm{V}_{\mathrm{c}}$ ratio of the autoxidation rates measured in the presence (Vs) and in the absence $(\mathrm{Vc})$ of sample. One SOD activity unit (U-525) has been defined as the activity which doubles the autoxidation background $\left(V_{s} / V_{c}=2\right)$. The equation which fits the standard curve is the same with all studied SODs. Another reagent with vinylpyridinium structure directly eliminates interferences due to sample mercaptans such as glutathione, by means of a very fast alkylation reaction. A fast, reproducible and specific measurement of SOD activity only requires a single determination per sample. At $\mathrm{pH}$ 8.8, an optimal assay sensitivity is achieved without inactivation of known SODs such as $\mathrm{Cu} / \mathrm{Zn}$-, $\mathrm{Mn}$ - or Fe-SOD.

\section{P8}

\section{Evaluation of efficiency of SOD entrapped into liposomes}

\section{S. Cote, ${ }^{1}$ E. Postaire, ${ }^{1}$ H. Fessi, ${ }^{1}$ G. Sarfati ${ }^{2}$ and $\mathbf{G}$. Hazebroucq ${ }^{1,2}$ \\ ${ }^{1}$ School of Pharmacy, Department of Clinical Pharmacy, 5, rue J. B. Clément, F-92290 Châtenay-Malabry, France; ${ }^{2}$ Hospital Cochin, rue du Fb St Jacques, F-75014 Paris, France}

Encapsulation of copper superoxide dismutase (SOD) in liposomes is a method performed to increase circulating half-life period and cell penetration of the enzyme.

Liposomes were prepared by controlled injection (Brevet CNRS 89401857-1) from dipalmitoylphosphatidylcholine, cholesterol, and stearylamine in a molar ratio of 14:7:4. Initially, enzyme to phospholipid ratio before controlled injection was $190 \mathrm{U}$ SOD $/ \mu \mathrm{mol}$ phospholipid.

Approximately, $40 \%$ of enzyme in aqueous solution was liposome associated with a variation in entrapment efficiency of $10 \%$.

The aim of our study consists of the comparison between the native SOD (bovine copper SOD) and the corresponding liposomal preparation. Two tests were carried out to evaluate enzyme efficiency.

Effects of native and liposomal SOD were studied with the help of an in vitro biological test (used to evaluate drugs with antiradicular activity). This test relies on the use of a water-soluble generator of free radicals $\left(2,2^{\prime}\right.$-azo-bis(2-amidinopropane) hydrochloride) which is incubated with human red blood cells at $37^{\circ} \mathrm{C}$ and the tested molecule: haemolysis was observed. According to our results, SOD does not present an antiradicular protective effect on red blood cells: superoxide anion radicals are not generated by this biological system.

On the other hand, incorporation of SOD into cationic liposomes greatly increases cell penetration and adsorption in comparison with the native form. With the use of human neutrophils as a cellular model, the intracellular SOD is quadrupled by concentrations of 2 and $4 \mathrm{U} / \mathrm{ml}$. Neutrophils are, partly, responsible for the initiation of the inflammation reaction due to superoxide anion production. Perhaps, a high level of associated SOD neutrophils could improve the activity of the enzyme in this phenomenon. Our next study will be to stimulate the neutrophil load in SOD and observe the effect on superoxide anion production.

\section{P9}

\section{New microtitration fluorometric assay for the evaluation of free radical scavengers}

\section{P. Rat, ${ }^{1,2}$ M. O. Christen, ${ }^{3}$ J.-M. Warnet ${ }^{2}$ and $M$. Adolphe ${ }^{1}$}

\begin{abstract}
${ }^{1}$ Laboratoire de Pharmacologie Cellulaire E.P.H.E-15 rue Ecole de Médecine F75006 Paris; ${ }^{2}$ Laboratoire de Toxicologie ( $\operatorname{Pr}$ J. R. CLAUDE) Faculté de pharmacie, F 75270 Paris Cedex 06; ${ }^{3}$ Laboratoires SOLVAY Pharma-L.T.M., B.P.22- F 92151 Suresnes, France
\end{abstract}

It has been recently shown that mitochondria are one of the first targets of free radicals induced by different drugs as paraquat, ethanol (Hirai, Inagaki 1992) or paracetamol (Burcham 91). Moreover mitochondrial activity can be easily evaluated with different specific fluorescent probes as Rodamine 123 with the Flow Cytometry method, but this is not well adapted to the screening of free radical scavengers. So, the aim of this study is to propose 
a new method to detect the fluorescence of Rh123 by microtitration as an alternative method.

For this purpose, different drugs were used: ethanol and paracetamol (AAP). These drugs were added to culture medium on L 929 or HepG2 cell line (human liver), associated or not with the antidote as $N$-acetylcysteine (NAC) or anethole dithiolthione (ADT). Mitochondrial alterations were assessed with rhodamine 123 ( $\mathrm{Rh} 123$ ). The stock solution $(20 \mathrm{mg} / \mathrm{ml})$ of $\mathrm{Rh} 123$ was diluted in culture medium and added to the culture for $30 \mathrm{~min}$, at $37^{\circ} \mathrm{C}$ in the dark. After extraction of the probe, the fluorescence (Exc.: $490 \mathrm{~nm}$, Em.: $530 \mathrm{~nm}$ ) can be measured with a new generation of fluorometric readers directly on a 96 well microplate. Furthermore, a high sensitivity was observed: $10^{-15} \mathrm{~g} / \mathrm{ml}$ of $\mathrm{Rh} 123$.

Only 24 h contact with AAP is sufficient to detect cell viability alteration with $\mathrm{Rh} 123$ compared with 48-72 h contact are necessary with Neutral Red assay. After 72 h-contact, cell viability was $49.9 \%$ vs. control with AAP $(150 \mu \mathrm{g} / \mathrm{ml})$ treated cell and $81 \%$ $v s$. control, with AAP + ADT $(12 \mu \mathrm{g} / \mathrm{ml})$ treated cells. So ADT can protect cells against AAP. The cytoprotective effect of ADT $(10 \mu \mathrm{M})$ against ethanol-induced free radical injury is easily detectable $(p<0.05)$ with all ethanol concentrations tested $(1-20 \%)$ with an optimum efficiency after $48 \mathrm{~h}$ contact.

In conclusion, $\mathrm{Rh} 123$ microtitration assay is a rapid quantitative method to evaluate, with a good sensitivity (Limit detection: $10^{-15} \mathrm{~g} / \mathrm{ml}$ of probe), cellular alterations. So it can be an alternative method to flow cytometry as a cell viability index and could be one of the first to signal free radical injury. These fluorometric methods can reveal more easily the cytoprotective effects and free radical scavenger properties of some drugs such as ADT.

\section{P10}

\section{The role of NADH in ethanol-induced microsomal lipid peroxidation}

\section{L. Mira, L. Barreira and C. F. Manso}

Instituto de Quimica Fisiológica, Faculdade de Medicina, 1600 Lisboa, Portugal

Lipid peroxidation is an oxygen free radical mediated process, which has been implicated in alcohol-induced liver injury. Ethanol metabolism may alter the $\mathrm{NAD}^{+} / \mathrm{NADH}$ ratio in favour of $\mathrm{NADH}$, and the oxidation of this nucleotide by aldehyde oxidase (A.O.), when dioxygen is the electron acceptor, produces $\mathrm{H}_{2} \mathrm{O}_{2}$ and $\mathrm{O}_{2}$.
Therefore studies were done to evaluate whether NADH oxidation by A.O. could promote lipid peroxidation. In vitro systems constituted by $\mathrm{NADH}$ and A.O. in the presence of ADP-FeIII catalysed lipid peroxidation of liver microsomes in a NADH and A.O. concentration-dependent manner.

When microsomes were incubated with a $\mathrm{NADH}$ generating system, consisting of ethanol, $\mathrm{NAD}^{+}$and alcohol dehydrogenase in the presence of ADP-FeIII and A.O. higher levels of lipid peroxidation were obtained.

The mechanisms responsible for lipid peroxidation induced by metabolites produced during ethanol metabolism, including the possible activation of a vitious cycle are discussed.

\section{P11}

\section{Structural modifications of human $\beta_{2}$ microglobulin treated with free radicals}

\section{Chantal Capeillere-Blandin, * Thierry Delaveau and Béatrice Descamps-Latscha}

INSERM U25, Clinique Néphrologique, Hôpital Necker, 161 Rue de Sèvres, 75743 Paris Cédex 15, France

*Present Address: UA 400, U.E.R. Biomédicale, 45 rue des Saints-Pères, 75270 Paris Cedex 06, France

Treatment of human $\beta_{2}$ microglobulin $\left(\beta_{2} \mathrm{~m}\right)$ with defined oxygen derived species generated radiolytically was studied ${ }^{1}$. Using SDS-polyacrylamide gel electrophoresis, the hydroxyl radicals $\left({ }^{\circ} \mathrm{OH}\right)$ caused the disappearance of the protein band at $12 \mathrm{kDa}$, specific for $\beta_{2} \mathrm{~m}$ and cross-linked the protein into protein bands stable to both SDS and reducing conditions. However, when ${ }^{\circ} \mathrm{OH}$ was generated under oxygen $\left(\mathrm{O}_{2}\right)$ in equimolar combination with the superoxide anion radical $\left(\mathrm{O}_{2}^{--}\right)$the high molecular weight protein products were less represented and fragmented derivatives were not obviously detectable. Exposure to ${ }^{\circ} \mathrm{OH}$ alone, or to $\mathrm{OH}+\mathrm{O}_{2}^{-}$in the presence of $\mathrm{O}_{2}$, induced the formation of $\beta_{2} \mathrm{~m}$ protein derivatives with more acidic net electrical charge. In contrast, $\mathrm{O}_{2}^{-}$alone was virtually inactive in both these respects. Changes in UV fluorescence during ${ }^{\circ} \mathrm{OH}$ attack revealed changes in conformation as confirmed by circular dichroism (CD) spectrometry. The high level of radicals caused the disappearance of the $\beta$-pleated sheet structure and the formation of random coil structure. Loss of tryptophan and significant production of dityrosine $\left(2,2^{\prime}\right.$-biphenol type) were noted, exhibiting a clear dose dependence with ${ }^{\circ} \mathrm{OH}$ alone or with ${ }^{\circ} \mathrm{OH}+\mathrm{O}_{2}^{-}$. The 
combination of ${ }^{\circ} \mathrm{OH}+\mathrm{O}_{2}^{-}$induced a pattern of changes very similar to that with ${ }^{\circ} \mathrm{OH}$ alone, more extensive for $\mathrm{CD}$ and tryptophan oxidation (two $\operatorname{Trp} / \beta_{2} \mathrm{~m}$ molecule), and more limited for dityrosine formation. Lower levels of these oxidative agents caused the formation of reproducible species at 18 and $25 \mathrm{kDa}$, recognised by antibodies against native $\beta_{2} \mathrm{~m}$. These findings would provide a model for the protein pattern observed in $\beta_{2} \mathrm{~m}$ amyloidosis described in the literature.

1. C. Capeillère-Blandin, T. Delaveau, B. Descamps-Lascha. Biochem J 1991; 277: 175-182.

\section{P12}

\section{Hypoxia/reoxygenation and inflammatory response of endothelium: role of oxygen-derived free radicals}

\section{O. Palluy, C. Bonne and G. Modat}

Laboratoire de Physiologie Cellulaire, Faculté de Pharmacie, Université Montpellier I, France

Reactive oxygen species (ROS) are known to cause various endothelial dysfunctions. In this study, we examined the role of hypoxia/reoxygenationinduced ROS on neutrophil adhesion and endothelial cytokine synthesis. Cultured human umbilical vein endothelial cells were submitted to $5 \mathrm{~h}$ hypoxia $\left(95 \% \mathrm{~N}_{2}, 5 \% \mathrm{CO}_{2}\right)$ followed by 20 min-24 h reoxygenation $\left(95 \%\right.$ air, $\left.5 \% \quad \mathrm{CO}_{2}\right)$. Adherence of resting neutrophils, added to monolayers for the last $15 \mathrm{~min}$ reoxygenation, was evaluated by myeloperoxidase assay. Interleukin-1 (IL-1) and interleukin-6 (IL-6) were measured in supernatants by specific enzyme immunoassays and bioassays respectively. Under these conditions, we found an increased adhesion of neutrophils with two peaks after $20 \mathrm{~min}$ and $4 \mathrm{~h}$ reoxygenation. This was correlated with the respective expression of the preformed granule membrane protein 140 (GMP140) and of the de novo synthetised endothelial leukocyte adhesion molecule-1 (ELAM-1) on endothelial surface. Otherwise, it was observed that there was a significant increase of IL-1 and IL-6 synthesis after $24 \mathrm{~h}$ reoxygenation. Addition of superoxide dismutase, catalase, or glutathione peroxidase to cultures before hypoxia efficiently prevented both neutrophil adhesion and cytokine synthesis. These results underline the crucial role played by endothelial-derived ROS in inflammatory and immunological events linked to ischaemia/ reperfusion processes. The protection offered by free radical scavengers emphasises the potential therapeutic use of antioxidants in post-ischaemic vascular disorders.
P13

\section{Hypoxia decreases the fibrinolytic potential of human endothelial cells: role of oxygen radicals}

\author{
O. Palluy, J. C. Gris, L. Merklein, C. Bonne \\ and G. Modat
}

Laboratoire de Physiologie Cellulaire, Faculté de Pharmacie, Université Montpellier I, France

Reactive oxygen species (ROS) produced in post-ischaemic periods are known to modulate endothelial functions including regulation of fibrinolysis. By contrast the role of ROS which could be generated by endothelium during ischaemia is still unclear. In this study, cultured human umbilical vein endothelial cells (HUVEC) were submitted to various times of hypoxia $(1-8 \mathrm{~h})$ as an in vitro model of ischaemia- and endothelial tissue-type plasminogen activator ( $t-\mathrm{PA}$ ) was measured by specific enzyme immunoassay. Hypoxia induced a time-dependent decrease in t-PA content in HUVEC supernatants reaching $72.3 \pm$ $1.1 \%$ after $8 \mathrm{~h}$. Addition of superoxide dismutase $(300 \mathrm{U} / \mathrm{ml})$ to cultures before hypoxia totally prevented this effect. Similar results were obtained with the xanthine oxidase inhibitor oxypurinol $(1 \mathrm{mM})$, thus strongly suggesting the participation of superoxide anion in t-PA modulation. The flavonoï rutin $(1 \mathrm{mM})$, an hydroxyl radical scavenger, was also (but less) protective and catalase (300 U/ml) was inefficient. These data demonstrate that ROS induced by hypoxia may contribute to decrease the fibrinolytic potential of endothelium.

\section{P14}

\section{Effect of deferoxamine on kidney lipoperoxidation induced by cold hypoxia/warm reoxygenation}

\section{S. Akritopoulos-Fourcroy, L. Mercklein, C. Bonne and G. Modat}

\section{Laboratoire de Physiologie Cellulaire, Faculté de Pharmacie, Université Montpellier I, France}

Oxygen-derived free radicals are an important cause of tissular injury in ischaemia/reperfusion processes among which organ transplantation. In this study we examined the effect of the iron chelator, deferoxamine on cold hypoxia/warm reoxygenationinduced peroxidation of rabbit kidney biopsies. Renal cortical slices were placed in the cold storage solution, Eurocollins for $24-72 \mathrm{~h}$ at $4^{\circ} \mathrm{C}$ and further incubated in M199 supplemented with $2 \%$ foetal 
calf serum under normoxic conditions (95\% air, 5\% $\mathrm{CO}_{2}$ ) for $24 \mathrm{~h}$ at $37^{\circ} \mathrm{C}$. Amounts of thiobarbituric acid reactive substances (TBARS) in disrupted slices were used as an index of lipid membrane peroxidation.

Cold hypoxia induced a time-dependent increase in TBARS which were furthermore significantly enhanced by reoxygenation. Addition of deferoxamine $\left(10^{-3} \mathrm{M}\right)$ to Eurocollins reduced TBARS formation not only during hypoxia $(\max .51 .5 \pm$ $2.16 \%$ after $72 \mathrm{~h}$ ) but also following reoxygenation $(44.09 \pm 6.76 \%)$.

Similar data were obtained with human kidney biopsies. The present results suggest the potential use of iron chelators in cold storage solutions for kidney transplants which could be thus preserved for longer periods with minor tissular alterations in the oxidative stress of reperfusion.

\section{P15 \\ Oxygen radicals adversly affect the regulation of vascular tone by nitric oxide in the rabbit retina}

\section{S. Vériac, ${ }^{1}$ G. Tissiée ${ }^{2}$ and C. Bonne ${ }^{1}$}

${ }^{1}$ Laboratoire de Physiologie Cellulaire, Faculté de Pharmacie, Université Montpellier I; ${ }^{2}$ Centre de Recherche, Laboratoire Chauvin, Montpellier, France

Choroidal blood flow was evaluated by the electrogenesis modulation in the rabbit retina. Flash electroretinograms (ERG) were recorded in the rabbit eye submitted to high intraocular pressure (HIOP) induced with a suction-cup. When intraocular pressure rose to $100 \mathrm{mmHg}$, ERG was suppressed because of blood flow arrest. ERG rapidly recovered even under HIOP when animals were pretreated either intravitreously with a nitric oxide donor, sodium nitroprusside, or intravenously with free radical scavengers, superoxide dismutase (SOD) + catalase. In contrast, injection of a nitric oxide synthesis inhibitor, nitro-Larginine, into the vitreous cavity inhibited the protective effect of SOD + catalase during HIOPinduced ERG extinction. These results suggest that nitric oxide could play a role in the regulation of ocular vessel tone and that severe ischaemia can impede this effect through oxygen-derived free radical generation.

\section{P16}

\section{Protective effect of moxisylyte from ischaemia and glutamate-induced neuronal damage in vitro}

\section{A. Muller, ${ }^{1}$ C. Cazevieille, ${ }^{1}$ D. Sincholle, ${ }^{2}$ P. Denis, ${ }^{3}$ L. Daumas ${ }^{3}$ and C. Bonne ${ }^{1}$}

${ }^{1}$ Laboratoire de Physiologie cellulaire, Université Montpellier I; ${ }^{2}$ CRID, Montpellier; ${ }^{3}$ Laboratories Sarget, Merignac, France

Moxisylyte (Carlytene ${ }^{\circledR}$ ) is an $\alpha_{1}$ adrenergic antagonist used for treatment of various diseases related to cerebral microcirculation deficiency.

In the present study we show that in addition, moxisylyte $\left(10^{-5}-10^{-3} \mathrm{M}\right)$ is able to protect cultured rat cortical neurons from the toxicity of glutamate $\left(10^{-5} \mathrm{M}\right)$ and from alterations induced by hypoxia/reoxygenation, i.e. lacticodehydrogenase release.

These effects could be explained by an antagonistic action of the drug to glutamate on its ionophoretic receptors, an effect which has been previously reported in electrophysiological studies (Jeantet et al. 1991).

Moreover, we show that moxisylyte is a potent scavenger of oxygen-derived free radicals and suggest that this property could contribute to the protective effect of the drug in the cultured neuron model of glutamate/oxidative stress.

\section{P17}

\section{Biochemical and pharmacological properties of SR 27388 , a dual antioxidant and PAF receptor antagonist}

\author{
J. M. Herbert, L. Fraisse, A. Bachy. \\ G. Valette, P. Savi and J. P. Maffrand
}

Sanofi Recherche, Toulouse, France

SR 27388 is a potent and competitive antagonist of the binding of $\left[{ }^{3} \mathrm{H}\right]-\mathrm{PAF}$ to its receptor on rabbit platelets exhibiting a $K_{\mathrm{i}}$ value of $10.5 \pm 1.2 \mathrm{nM}$. SR 27388 potently inhibited PAF-induced aggregation of rabbit platelets in vitro $\left(\mathrm{IC}_{50}=65 \pm 12 \mathrm{nM}\right)$ and had no effect on the action of other platelet aggregating agents. SR 27388 prevented in a dose-dependent manner the formation of thiobarbituric acid reactive substances during membrane peroxidation $\left(\mathrm{IC}_{50}=0.7 \mu \mathrm{M}\right)$ and inhibited reduc- 
tion of the stable 1,1-diphenyl-2-picrylhydrazyl radical, indicating that the antioxidant potency of SR 27388 was due to an efficient radical scavenging activity. SR 27388 displayed marked in vitro inhibition of zymosan-induced oxidative burst in human monuclear cells $\left(\mathrm{IC}_{50}=3 \mu \mathrm{M}\right)$.

In vivo, SR 27388 protected mice from $100 \mu \mathrm{g} / \mathrm{kg}$ PAF-induced death with an $\mathrm{ED}_{50}$ value of $500 \mu \mathrm{g} / \mathrm{kg}$, when given i.v., $5 \mathrm{~min}$ before PAF challenge or p.o. $\left(\mathrm{ED}_{50}=800 \mu \mathrm{g} / \mathrm{kg}\right)$ when given $1 \mathrm{~h}$ before PAF administration. Similarly, i.v. or oral doses of SR 27388 afforded in mice complete protection against endotoxin-induced lethality $\left(\mathrm{ED}_{50}\right.$ values were $250 \mu \mathrm{g} / \mathrm{kg}$ and $1.3 \mathrm{mg} / \mathrm{kg}$ respectively). In ovalbumin-presensibilised rabbits, SR 27388 premixed with the allergen inhibited in a dose-dependent manner allergen-induced oedema formation in the skin $\left(\mathrm{ED}_{50}=0.1 \mu \mathrm{mole} / \mathrm{site}\right)$. After an i.v. administration of $10 \mathrm{mg} / \mathrm{kg}$, SR 27388 significantly protected mice against alloxan-induced diabetes.

These results show that SR 27388 is a potent and orally active dual antioxidant and PAF receptor antagonist.

\section{P18}

\section{Aerosol administration of antigen and substance $P$ induces superoxide anion production by guinea-pig alveolar macrophages}

\section{E. Boichot, ${ }^{*}$ V. Lagente, B. Arbeille, ${ }^{1}$ J.-M. Mencia-Huerta and P. Braquet}

Institut Henri Beaufour, 91952 Les Ulis, France:

${ }^{1}$ Faculté de Médecine, Unité de

Microscopie électronique, 37032 Tours, France

*Present Address: Bio-Inova Research Laboratory, 78370 Plaisir, France

Alveolar macrophages (AM) are resident cells of the bronchopulmonary system that may participate to the airway alterations during allergic reactions. We previously demonstrated that an enhanced bronchopulmonary response (BR) was observed after ovalbumine (OA) challenge in sensitised guineapigs and following aerosolised substance $\mathrm{P}(\mathrm{SP})$ in phosphoramidon-pretreated guinea-pigs. We now investigated the production of superoxide anion by AM after OA challenge and after aerosol administration of substance $\mathrm{P}$ in guinea-pigs. Hartley guinea-pigs were sensitised by $30 \mathrm{~min}$ two aerosol exposures at a $48 \mathrm{~h}$-interval to a saline solution containing $0.2 \%$ OA. Fifteen to 20 days after, guinea-pigs were challenged by aerosolised OA or saline alone. Guinea-pig AM were obtained by five bronchoalveolar lavages with $5 \mathrm{ml}$ saline solution $\left(37^{\circ} \mathrm{C}\right)$. As determined by electron microscopy, AM from sensitised guinea-pigs exposed to OA were characterised by a marked phagocytic activity as compared to AM from control animals. Production of superoxide anions from AM was measured by the luminol-dependent chemiluminescence technique (LDCL) using a computerised chemiluminometer (Berthold LB $9505 \mathrm{C})$. Platelet activating factor (PAF) $(0.01 \mu \mathrm{M}$ or $1 \mu \mathrm{M})$, or the tripeptide, $N$-formyl-methionylleucyl-phenylalanine (f-MLP) $(0.01 \mu \mathrm{M}$ or $1 \mu \mathrm{M})$, induced a marked increase in LDCL from AM. This phenomenon was significantly reduced when the cells were incubated in the presence of superoxide dismutase $(30 \mathrm{U} / \mathrm{ml})$, suggesting that it was mainly due to the generation of superoxide anions. AM from sensitised guinea-pigs challenged by aerosol with OA exhibited an increased in LDCL when the cells were stimulated in vitro with PAF $(0.01 \mu \mathrm{M}$ or $1 \mu \mathrm{M})$ or $\mathrm{f}-\mathrm{MLP}(1 \mu \mathrm{M})$, as compared to those from control animals. Exposure of non-sensitised guineapigs to SP $(1 \mathrm{mg} / \mathrm{ml})$ induced after $24 \mathrm{~h}$ a small increase in the f-MLP- $(0.01 \mu \mathrm{M}$ and $1 \mu \mathrm{M})$ and PAF- $(0.01 \mu \mathrm{M})$ induced superoxide anion production from AM. In addition, when phosphoramidon(0.1 $\mathrm{mM}$ for $15 \mathrm{~min})$ pretreated guinea-pigs were exposed to $\mathrm{SP}(0.1 \mathrm{mg} / \mathrm{ml})$ a marked and significant increase in superoxide anion production from AM stimulated by f-MLP and, in a lower extent by PAF, was observed. The enhanced reactivity of alveolar macrophages, as demonstrated by the increase in superoxide anion production, could participate to the bronchial hyperresponsiveness observed after OA challenge and aerosol administration of SP in the guinea-pigs.

P19

\section{Reactive oxygen species released by human blood leukocytes from asthmatic patients; comparison with healthy subjects}

\section{Vachier, Ch. Le Doucen, P. Chanez, Ph. Godard and M. Damon}

INSERM U58, Clinique des Maladies Respiratoires, Montpellier, France

Inflammatory process in asthma is characterised by an infiltration of blood cells such as polymorpho- 
nuclear and mononuclear cells. These cells were known to be activated and to release numerous mediators. The aim of this study was to determine the activation state of blood cells related to the stage of asthma through their capacity to release ROS. Nine healthy subjects (HS) and 14 patients with asthma were studied. Six patients were in stable state (SA), eight developed an asthma attack (AA) $24 \mathrm{~h}$ before taking of blood sampling. Polymorphonuclear neutrophils (PMN) were separated over a discontinuous Percoll gradient. Monocytes (MO) were purified by adherence and lymphocytes (Ly) recovered in medium. The cells were studied and after stimulation by phorbol myristate acetate (PMA, $10^{-7} \mathrm{M}$ ). The release of ROS was determined by chemiluminescence (CL) using lucigenin $\left(\mathrm{O}_{2}^{-}\right)$and luminol (the whole set of ROS) with or without horseradish peroxidase as luminescent probes. Results were analysed at CL peak and expressed as $\mathrm{mV} / 5 \times 10^{5}$ cells. Non-stimulated PMN, MO and Ly from AA released higher quantities of $\mathrm{O}_{2}^{-}$than $\mathrm{SA}$ and HS. After stimulation no significant difference was observed excepted for Ly. When luminol was used, spontaneous release of ROS was higher in PMN from AA. After stimulation, in the three populations, the responses were higher in AA than in HS and SA. In the presence of HRP, the spontaneous release of ROS was similar to that observed without HRP. But after stimulation, $\mathrm{MO}$ and Ly responses were similar with higher values for AA. PMN responses were identical to those observed with luminol alone: $\mathrm{AA}>\mathrm{HS}>\mathrm{SA}$. The in vitro reactivity of blood cells led to consider that just after an asthma attack blood cells remained activated during at least $24 \mathrm{~h}$ as compared to stable state.
P20

\section{Oxidative Stress Status (OSS) in HIV seropositive patients. Possible improvement by antioxidant therapy}

\author{
J. Emerit, ${ }^{1}$ D. Bonnefont, ${ }^{2}$ M. C. Jaudon ${ }^{2}$ \\ and 0 . Lopez
}

\author{
${ }^{1}$ Service de Médecine Interne, La Salpétrière, \\ Paris; ${ }^{2}$ Laboratoire de Biochimie, La Salpétrière, \\ Paris, France
}

A growing body of evidence concurs to an antioxidant deficiency in HIV seropositive patients. In cultured cells oxidative stress increases HIV replication and antioxidants decrease it. Vitamin $\mathrm{E}$ improves the antiviral action of AZT. We studied: the action of dithiocarbamate (D'TC) $v$ s. placebo on OSS in $29 \mathrm{HIV}$ seropositive patients; the action of antioxidants therapy with several antioxidants including superoxide dismutase (SOD) in three HIV seropositive patients, also treated with AZT.

After 2 years of DTC administration, there was no difference in OSS between treated (16) and placebo (13) groups. The clinical evolution in the two groups was also the same. In three patients treated with several antioxidants (Vit E, Vit A, selenium, $N$-acetyl cysteine) and $\mathrm{Cu} \mathrm{Zn}$ SOD the OSS improved well as the biological markers monitored (CD4, immunoglobulin, $\beta_{2}$-microglobulin). One patient returned to work for 1 year after a 1 year interruption in very good health.

The antioxidant therapy associated with antiviral treatment deserves to be tested in controlled trial monitored by OSS and biological markers of HIV infection. 


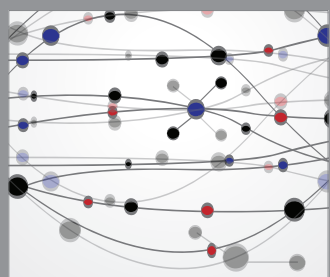

The Scientific World Journal
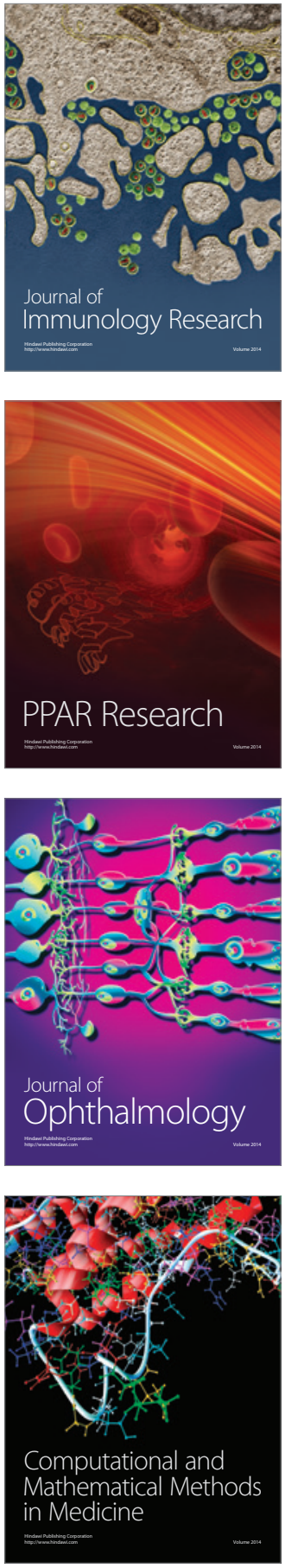

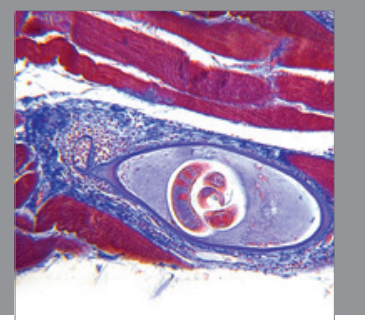

Gastroenterology

Research and Practice
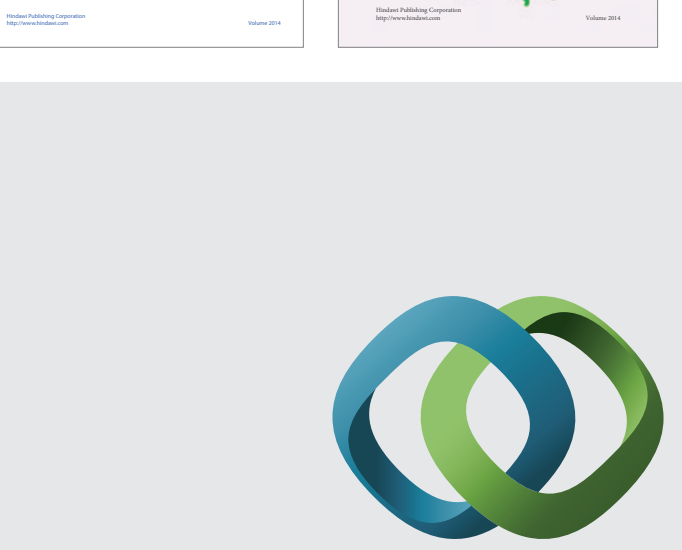

\section{Hindawi}

Submit your manuscripts at

http://www.hindawi.com
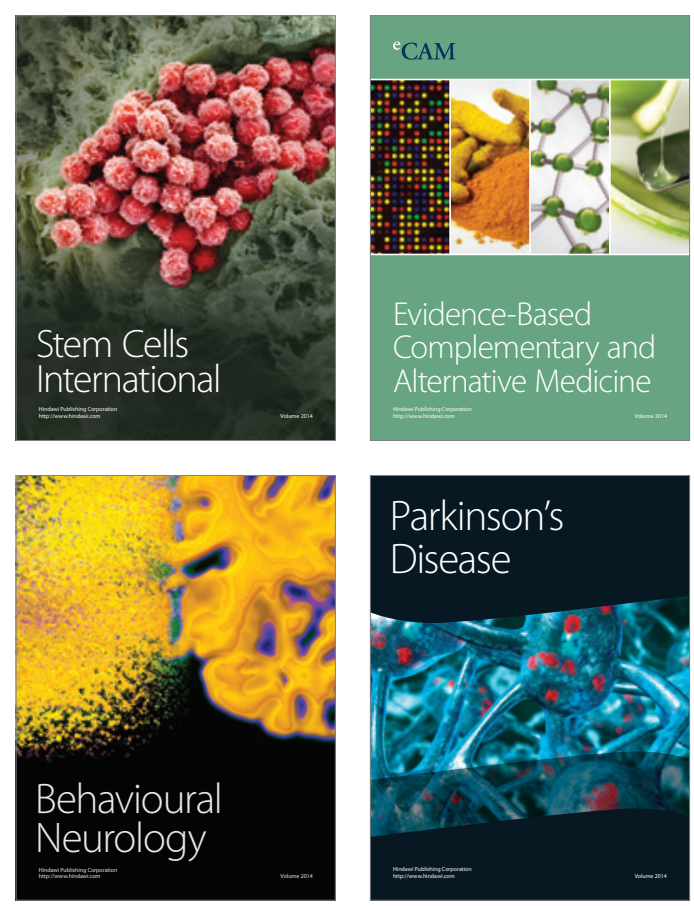

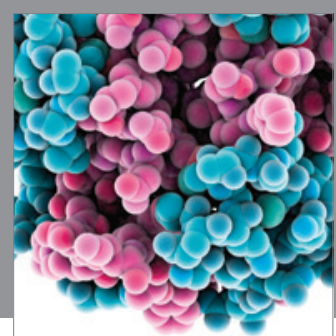

Journal of
Diabetes Research

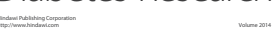

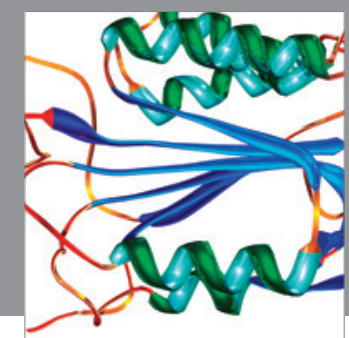

Disease Markers
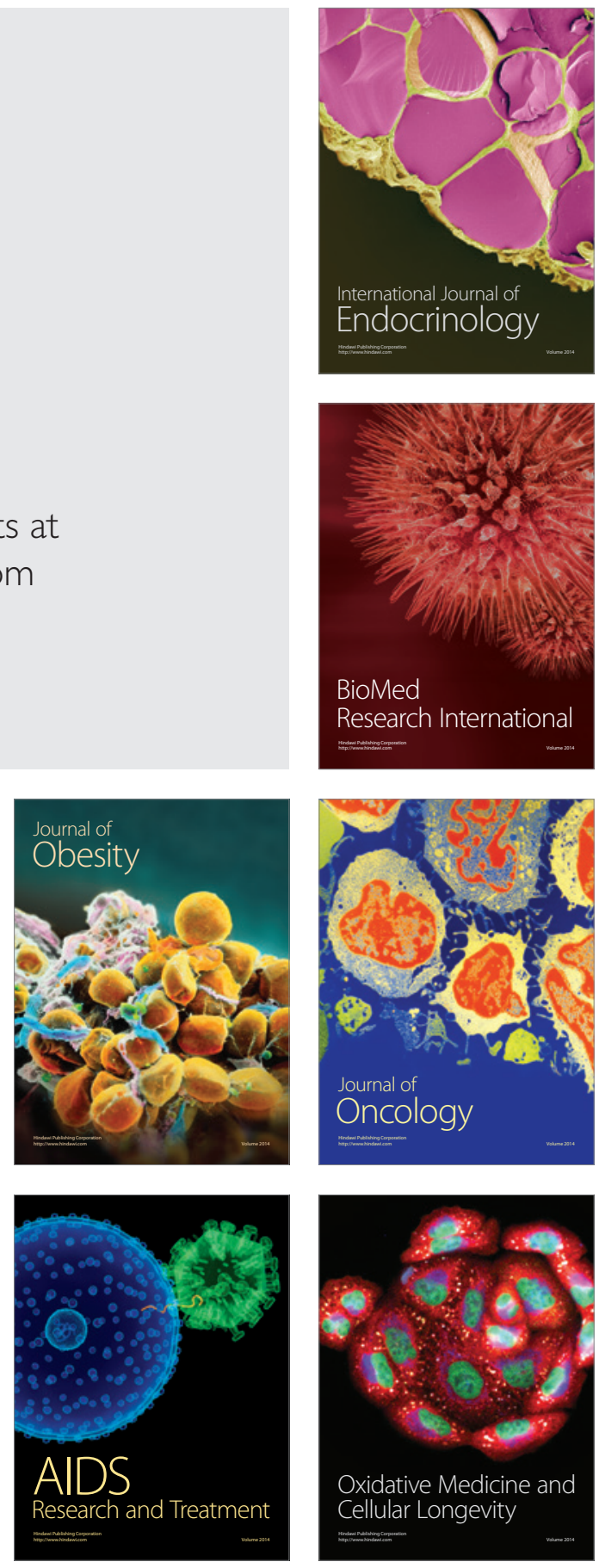